\title{
Comparison of different prostatic markers in lymph node and distant metastases of prostate cancer
}

\author{
Angela Queisser ${ }^{1,3}$, Susanne A Hagedorn ${ }^{1,3}$, Martin Braun ${ }^{1}$, Wenzel Vogel ${ }^{1}$, Stefan \\ Duensing $^{2}$ and Sven Perner ${ }^{1}$ \\ ${ }^{1}$ Department of Prostate Cancer Research, Institute of Pathology, University Hospital of Bonn and Centre \\ for Integrated Oncology (CIO) Cologne-Bonn, Bonn, Germany and ${ }^{2}$ Section of Molecular Urooncology, \\ Department of Urology, University of Heidelberg School of Medicine, Heidelberg, Germany
}

\begin{abstract}
Prostate cancer is mostly diagnosed at an early stage; however, some tumors are diagnosed in a metastatic stage as cancer of unknown primary origin. In order to allow specific treatment in the case of prostate cancer presenting as cancer of unknown primary origin, it is important to determine the tumor origin. Prostate-specific antigen is used as a diagnostic marker for prostate cancer but the expression declines with progression to castration-resistant prostate cancer. Aim of this study was to identify the most informative marker constellation, which is able to detect metastatic prostate cancer at high sensitivity. The widely used prostate cancer markers such as prostate-specific antigen, prostate-specific acid phosphatase, androgen receptor, prostate-specific membrane antigen, prostein, and ETS-related gene were investigated for their sensitivity to detect prostatic origin of metastases. Expression of prostate-specific antigen, prostate-specific acid phosphatase, androgen receptor, prostate-specific membrane antigen, prostein, and ETS-related gene was determined on archived tissue specimens consisting of benign prostatic tissue $(n=9)$, primary prostate cancer $(n=79)$, lymph node metastases $(n=58)$, and distant metastases $(n=39)$ using immunohistochemistry. The staining intensity was categorized as negative (0), weak (1), moderate (2), and strong (3). All markers except ETS-related gene were able to detect at least $70 \%$ of lymph node metastases and distant metastases, with prostate-specific antigen, androgen receptor, and prostate-specific membrane antigen having the highest sensitivity $(97 \%, 91 \%$, and $94 \%$, respectively). A further increase of the sensitivity up to $98 \%$ and $100 \%$ could be achieved by the combination of prostate-specific antigen, prostate-specific membrane antigen, or androgen receptor for lymph node metastases and for distant metastases, respectively. The same sensitivity could be reached by combining prostate-specific membrane antigen and prostein. Our data show that a combined staining of at least two prostate markers should be utilized to identify metastases as originating from prostate cancer.

Modern Pathology (2015) 28, 138-145; doi:10.1038/modpathol.2014.77; published online 13 June 2014
\end{abstract}

Prostate cancer is the most commonly diagnosed non-epidermal cancer and second-most common cancer-related cause of death in men in the western world. ${ }^{1}$ The patients' death is often caused by the development of castration-resistant prostate cancer. Most prostate cancers are detected early in a localized stage; however, in some cases, metastases

Correspondence: Professor S Perner, MD, PhD, Department of Prostate Cancer Research, Institute of Pathology and Centre for Integrated Oncology (CIO) Cologne-Bonn, University Hospital of Bonn, Sigmund-Freud Strasse 25, 53127 Bonn, Germany.

E-mail: sven.perner1972@gmail.com

${ }^{3}$ These authors contributed equally to this work.

Received 11 February 2014; accepted 17 April 2014; published online 13 June 2014 can be the first manifestation of a prostate cancer. ${ }^{2}$ Unlike other carcinomas, hormone-sensitive prostate cancer responds to hormonal therapy and therefore the identification of the tumor origin is important in order to allow specific treatment. Male patients with a metastatic adenocarcinoma are routinely assessed for prostatic origin using prostate-specific immunohistochemistry markers like prostate-specific antigen (PSA). ${ }^{3}$ PSA is highly expressed in benign prostate tissue as well as in the large majority of prostatic adenocarcinomas. ${ }^{4}$ However, recent studies found a loss of PSA expression in distant metastases in $10-20 \%$ of the investigated cases. ${ }^{2,4}$ Moreover, the specificity of PSA immunohistochemistry is limited, as immunoreactivity for PSA has additionally been detected in some non-prostatic tissues., 
Nevertheless, PSA is the only marker recommended by the guidelines to show prostatic origin of a metastatic lesion. ${ }^{3}$

It has been demonstrated that even some cases of primary prostate cancer were negative for PSA but could be detected by prostate-specific acid phosphatase (PSAP) and thus might be a good additional marker. ${ }^{7,8}$ In contrast to PSA, prostate-specific membrane antigen (PSMA) and prostein (also known as p501s or SLC45A3) were recently described to be highly specific for prostatic tissue., ${ }^{9,10}$ Since TMPRSS2-ERG rearrangements, leading to an ETSrelated gene (ERG) overexpression, ${ }^{11}$ are amongst the most common and disease-specific genetic alterations in locally defined prostate cancer, ${ }^{12-14}$ ERG might be of diagnostic potential. While TMPRSS2$E R G$ is an early event in prostate cancer, ${ }^{11,15}$ androgen receptor (AR) amplifications leading to an AR overexpression are linked to advanced prostate cancer stages as well as hormone-refractory metastases. ${ }^{16,17}$ While these above-mentioned markers are mainly investigated in primary prostate cancer, studies including metastatic prostate cancer are rare or absent. Hence, the aim of this study was to identify informative tissue markers, which are able to detect advanced metastatic stages of prostate cancer and to investigate their use for diagnostic applications. Therefore, we studied the expression of the commonly used markers PSA, PSAP, AR, PSMA, prostein and ERG in a series of primary prostate cancer, lymph node metastases, and distant metastases.

\section{Materials and methods}

\section{Cohort}

Approved by the ethics committee of the University of Bonn, we studied a prostate cancer progression cohort containing nine benign prostate samples, 79 primary prostate cancer samples, 58 lymph node metastases, and 39 distant metastases of prostate cancer samples, primarily of osseous origin. In $43 \%$ of the investigated lymph node metastases, the corresponding primary tumor was included. In seven cases of primary tumors, 2-5 corresponding lymph node metastases were investigated. The remaining lymph node metastases do not relate to primary tumors. The cohort contains tumor material from Middle European who consecutively underwent radical prostatectomy. The regional lymph node metastases were extracted in the context of the radical prostatectomy. Metastases were diagnosed as originating from the prostate prior to the study and treated with hormone ablation. All patients were diagnosed and treated at the University Hospital of Bonn.

\section{Tissue Microarray Construction}

Immunohistochemical experiments were performed on tissue microarrays. Tissue microarrays were constructed as described previously. ${ }^{12}$ All prostatic specimens from osseous origin were routinely decalcified by using the chelating agent EDTA. Formalin-fixed paraffin-embedded tissues were cut in $4-\mu \mathrm{m}$ thick sections and mounted on slides. After staining with hematoxylin and eosin, relevant areas of normal prostatic tissue, primary prostate cancer, localized lymph node, and distant metastases were determined and circled by a pathologist (SP). Three representative cores of the circled regions measuring $0.6 \mathrm{~mm}$ in diameter from each formalin-fixed paraffin-embedded benign tissue, primary tumor, lymph node metastasis, and distant metastasis (donor blocks) were assembled into tissue microarray blocks (recipient blocks) using a semiautomatic tissue arrayer (Beecher Instruments, Sun Prairie, WI, USA). Hematoxylin and eosin-stained tissue microarray sections were assessed again to confirm the histology of the selected regions.

\section{Immunohistochemistry}

Immunohistochemical staining was conducted using the Ventana Benchmark automated staining system (Ventana Medical System, Tuscon, AZ, USA) as decribed previously. ${ }^{11}$ In brief, slides were incubated with the primary antibody (DAKO, Hamburg, Germany and BioLogo, Kronshagen, Germany) (Table 1) for 30 min at room temperature. Antibody dilution was performed using a Ventana diluent. Signal detection was performed using the UltraView-DAB detection kit (Ventana Medical System, Tuscon, AZ, USA). Slides were counterstained with hematoxylin, dehydrated, and mounted.

Immunohistochemical stains were independently evaluated by two pathologists (MB, SP). Only cases with at least one assessable core were included in this analysis. Staining was assessed as positive for PSMA if the staining was apical, for AR and ERG if the staining was nuclear, and for PSA, PSAP, and prostein if the staining was cytoplasmic. Staining intensity was quantified as follows: 0 as negative, 1 as weak, 2 as moderate, and 3 as strong. Samples with a lack of tissue or absence of carcinoma were excluded.

\section{Statistics}

The expression intensities in the different specimens were analyzed using Student's $t$-test. Statistics

Table 1 Antibody dilution and pretreatment

\begin{tabular}{lllcl}
\hline Antibody & Clone & Company & Dilution & Pretreatment \\
\hline AR & AR441 & DAKO & $1: 400$ & Citrate pH 6 \\
ERG & EPR3864 & BioLogo & $1: 100$ & Citrate pH 6 \\
PSA & Polyclonal & DAKO & $1: 20000$ & Without \\
PSAP & Polyclonal & DAKO & $1: 6000$ & Without \\
PSMA & 3E6 & DAKO & $1: 500$ & Citrate pH 6 \\
Prostein & 10E3 & DAKO & $1: 100$ & Tris/EDTA pH 8 \\
\hline
\end{tabular}


were performed using SPSS Statistics 19 (SPSS, Chicago, IL, USA) with a significance level of $\leq 0.05$.

\section{Results}

To identify the most informative marker or marker constellation, which is able to identify the prostatic origin of a cancer of unknown primary, immunohistochemical analyses using antibodies against PSA, PSAP, AR, PSMA, prostein, and ERG were performed on a prostate cancer progression cohort consisting of 58 lymph node metastases and 39 distant metastases. As the markers are well established on primary prostate cancer as well as normal prostatic tissue, we included nine benign prostatic tissues and 79 primary prostate cancers as a control.

For PSA, a typical cytoplasmic expression could be detected in normal prostate glands as well as in primary prostate cancer and advanced prostate cancer (Figures 1a-d). All benign prostatic tissues of our cohort expressed PSA. Furthermore, 96\% of primary prostate cancer samples were positive for PSA as well as $89 \%$ and $97 \%$ of lymph node metastases and distant metastases, respectively (Table 2). However, the staining intensity declined significantly in neoplastic tissue compared with benign prostatic tissue $(P<0.001)$ (Figure 2a).

PSAP expression was also detected in the cytoplasm of benign prostatic tissue as well as in primary prostate cancer, lymph node metastases, and distant metastases (Figures 1e-h). In total, 100\% of benign prostatic tissue and $95 \%$ of primary prostate cancer showed a PSAP expression (Table 2). The number of PSAP-positive cases of lymph node metastases and distant metastases decreased to $84 \%$ and $77 \%$, respectively. Moreover, the intensity of the staining was significantly reduced in lymph node metastases $(P=0.034)$ as well as distant metastases $(P<0.001)$ compared with primary prostate cancer (Figures 1e-h and $2 \mathrm{~b}$ ).

Nuclear AR expression was detected in $44 \%$ of benign prostatic tissue, $82 \%$ of primary prostate cancer cases, $74 \%$ of lymph node metastases cases; 91\% of distant metastases cases displayed a positive expression for AR (Figures 1i-l, Table 2). AR was only expressed weakly in benign prostatic tissue. We could detect a significantly increased intensity of the AR expression in metastatic prostate cancer compared with benign prostatic tissue $(P<0.001)$ (Figure 2c).

PSMA showed a predominantly apical staining in benign prostatic tissue and malignant tissue (Figures $1 \mathrm{~m}-\mathrm{p})$. Some cases of our cohort additionally expressed PSMA in the cytoplasm (data not shown). While primary prostate cancer was positive in $87 \%$ of the cases, lymph node metastases and distant metastases showed a PSMA expression in 91\% and 94\% cases, respectively (Table 2). PSMA was moderately expressed in benign prostatic tissue but was significantly higher expressed in primary prostate cancer $(P=0.003)$. Furthermore, the staining intensity of PMSA was significantly higher in lymph node metastases $(P=0.007)$ and distant metastases $(P=0.003)$ compared with primary prostate cancer (Figure 2d).

In benign prostatic luminal epithelia, prostein showed a granular cytoplasmic staining consistent with the known Golgi distribution (Figure 1q), whereas stromal cells and basal cells were negative. Prostein was expressed in $100 \%$ of benign prostatic tissue and $96 \%$ of primary prostate cancer. The expression rate of prostein was lower in metastatic prostate cancer, with $81 \%$ of lymph node metastases and $89 \%$ of distant metastases (Figures 1r-t, Table 2). Furthermore, we found a significantly lower expression in malignant compared with benign tissue (Figure 2e). The mean intensity was lower in metastases as compared with primary prostate cancer; however, this difference did not reach significance $(P=0.07)$.

All the investigated benign prostatic tissues were negative for ERG. In contrast, we found a nuclear ERG expression in primary prostate cancer at a frequency of $53 \%$. ERG was expressed in $53 \%$ of the lymph node metastases and in $30 \%$ of the distant metastases (Figures $1 \mathrm{u}-\mathrm{x}$ and Table 2). Moreover, the staining intensity did not differ between primary prostate cancer and metastatic prostate cancer (Figure 2f).

In summary, PSA, AR, and PSMA had the highest sensitivities (97\%, 91\%, and 94\%, respectively) in the investigated distant metastases compared with ERG $(30 \%)$, prostein $(89 \%)$, and PSAP $(77 \%)$. Lymph node and distant metastases negative for PSA could be detected either by AR or prostein. Using a combination of PSA with either AR or prostein or a panel of PSMA and prostein could further increase the sensitivity up to $98 \%$ for lymph node metastases and $100 \%$ for distant metastases. Only one lymph node metastasis of our cohort could not be detected by any marker.

\section{Discussion}

The prostate is often included in the list of possible primary sites of metastatic adenocarcinomas. ${ }^{18} \mathrm{In}$ cases of cancer of unknown primary origin, male patients are routinely assessed for the expression of prostatic markers to determine whether the origin of a metastasis is prostatic. ${ }^{19}$ Widely used markers such as PSA, PSAP, AR, PSMA, prostein, and ERG are well examined in primary prostate cancer but not in metastases. Due to molecular alterations in prostate cancer progression, expression of the used markers in metastatic prostate cancer can differ from primary prostate cancer.

PSA is highly expressed in benign prostatic tissue as well as in the large majority of primary prostatic adenocarcinomas. ${ }^{4}$ Though PSA is the only marker recommended in cancer of unknown primary guidelines to identify prostate cancer, studies in 

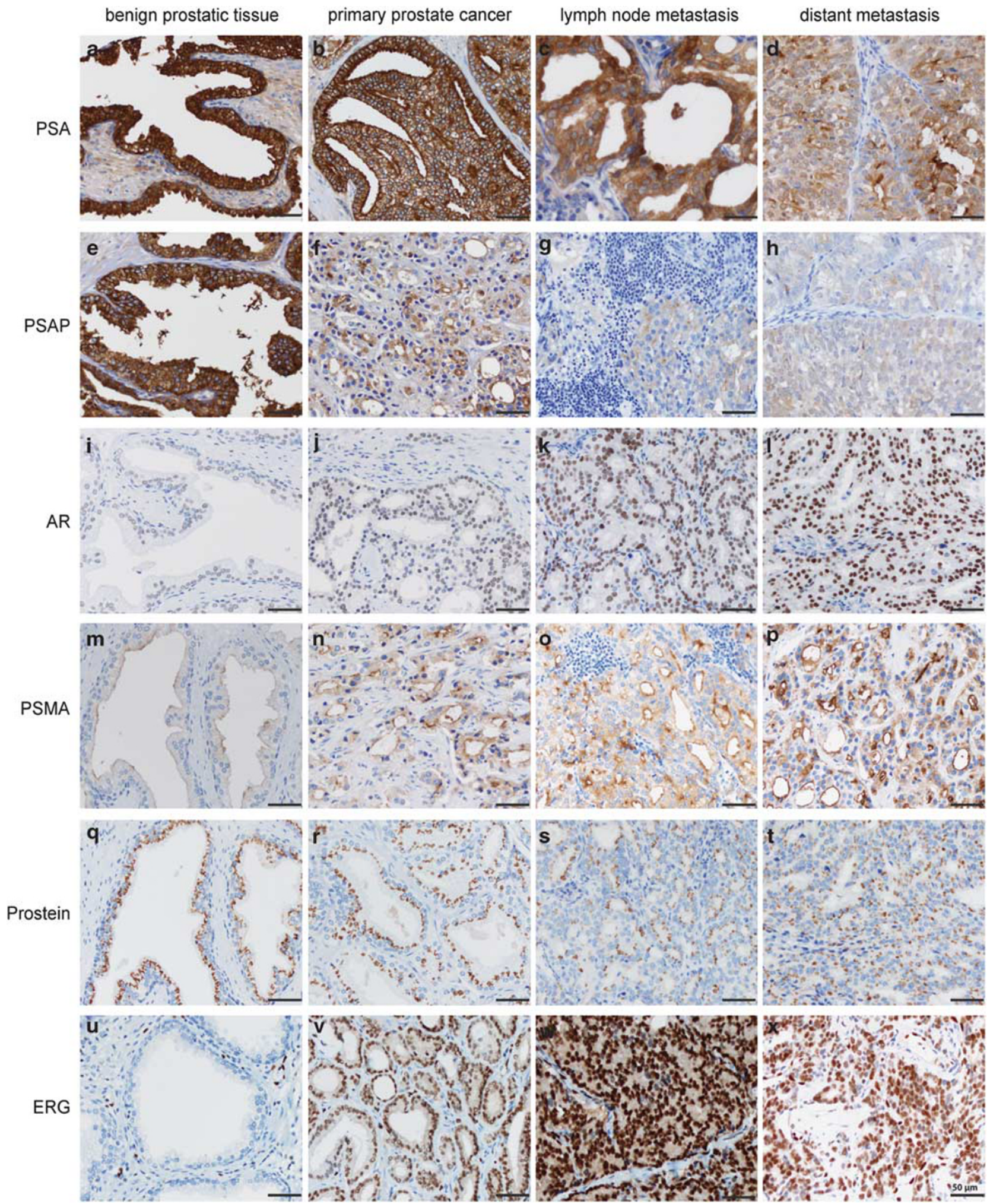

Figure 1 Representative immunohistochemical images of PSA, PSAP, AR, PSMA, prostein, and ERG in benign and neoplastic prostatic tissue. PSA is expressed in the cytoplasm of benign prostatic tissue (a), primary prostate cancer (b), lymph node metastases (c), and distant metastases (d). PSAP is expressed in the cytoplasm of benign prostatic tissue (e), primary prostate cancer (f), lymph node metastases (g), and distant metastases (h). AR is expressed weakly in the nucleus of benign prostatic tissue (i) and strongly in the nucleus of primary prostate cancers (j), lymph node metastases (k), and distant metastases (l). PSMA is expressed in the cytoplasm with a mainly apical pattern in benign prostatic tissue $(\mathbf{m})$, primary prostate cancer (n), lymph node metastases (o), and distant metastases (p). Prostein is expressed in the cytoplasm with Golgi-type distribution in benign prostatic tissue (q), primary prostate cancer (r), lymph node (s), and distant metastases (t). ERG is not expressed in the nucleus of benign prostatic tissue (u) but in primary prostate cancer (v), lymph node metastases $(\mathbf{w})$, and distant metastases $(\mathbf{x})$. 
Table 2 PSA, PSAP, AR, PSMA, prostein, and ERG expression in benign prostatic tissue, primary prostate cancer, lymph node metastases, and distant metastases

\begin{tabular}{|c|c|c|c|c|c|c|c|c|c|c|c|c|}
\hline & \multicolumn{2}{|c|}{$P S A$} & \multicolumn{2}{|c|}{$P S A P$} & \multicolumn{2}{|c|}{$A R$} & \multicolumn{2}{|c|}{$P S M A$} & \multicolumn{2}{|c|}{ Prostein } & \multicolumn{2}{|c|}{$E R G$} \\
\hline & $\begin{array}{l}\text { Positive } \\
\mathrm{n}(\%)\end{array}$ & $\begin{array}{c}\text { Total } \\
\mathrm{n}\end{array}$ & $\begin{array}{l}\text { Positive } \\
\text { n (\%) }\end{array}$ & $\begin{array}{c}\text { Total } \\
\mathrm{n}\end{array}$ & $\begin{array}{l}\text { Positive } \\
\text { n (\%) }\end{array}$ & $\begin{array}{c}\text { Total } \\
\mathrm{n}\end{array}$ & $\begin{array}{l}\text { Positive } \\
\text { n (\%) }\end{array}$ & $\begin{array}{c}\text { Total } \\
\mathrm{n}\end{array}$ & $\begin{array}{l}\text { Positive } \\
\text { n (\%) }\end{array}$ & $\begin{array}{c}\text { Total } \\
\mathrm{n}\end{array}$ & $\begin{array}{l}\text { Positive } \\
\text { n (\%) }\end{array}$ & $\begin{array}{c}\text { Total } \\
\mathrm{n}\end{array}$ \\
\hline $\begin{array}{l}\text { Benign prostatic } \\
\text { tissue }\end{array}$ & $9(100)$ & 9 & $9(100)$ & 9 & $4(44)$ & 9 & $9(100)$ & 9 & $9(100)$ & 9 & $0(0)$ & 9 \\
\hline $\begin{array}{l}\text { Primary prostate } \\
\text { cancer }\end{array}$ & $76(96)$ & 79 & 75 (95) & 79 & $65(82)$ & 79 & $69(87)$ & 79 & $76(96)$ & 79 & $41(53)$ & 78 \\
\hline $\begin{array}{l}\text { Lymph node } \\
\text { metastases }\end{array}$ & $51(89)$ & 57 & $48(84)$ & 57 & $42(74)$ & 57 & $52(91)$ & 57 & $46(81)$ & 57 & $30(53)$ & 57 \\
\hline Distant metastases & $36(97)$ & 37 & $27(77)$ & 35 & $32(91)$ & 35 & $33(94)$ & 35 & $32(89)$ & 36 & $10(30)$ & 33 \\
\hline
\end{tabular}

Abbreviations: AR, androgen receptor; ERG, ETS-related gene; PSA, prostate-specific antigen; PSAP, prostate-specific acid phosphatase; PSMA, prostate-specific membrane antigen.

distant metastases concerning PSA expression are rare. Two studies showed that a loss of PSA expression in metastatic prostate cancer occurs in approximately $15-20 \%$ of the cases. ${ }^{2,4}$ In contrast to these findings, we obtained a higher sensitivity of $89 \%$ for lymph node and $97 \%$ for distant metastases for PSA. Moreover, we could confirm that PSA was strongly expressed in normal tissue but expression intensity was lower in neoplastic prostatic tissue. ${ }^{2,20}$

PSAP was the first human tumor marker ever described in prostate cancer. ${ }^{21}$ Goldstein ${ }^{19}$ showed a decreased PSAP immunoreactivity in prostate cancers with higher Gleason score. Kirschenbaum et $a 1^{18}$ investigated PSAP in bone metastases of prostate cancer and found a strong expression in all the eight cases. In contrast, $16-23 \%$ of our metastases showed no expression of PSAP. Moreover, we demonstrated a significant decrease of expression from primary to metastatic prostate cancer. We observed a weak staining for PSAP in $31 \%$ of lymph node metastases and in $52 \%$ of distant metastases of the positive cases. Weak staining can be difficult to distinguish from artefacts and thus increases the risk of misinterpretation. Therefore we conclude that PSAP should not be the first choice for diagnostic work-up.

Amplification of AR gene is common in distant metastases of prostate cancer and leads to a higher AR protein expression. ${ }^{22,23}$ Since AR overexpression is a late event in the progression of prostate cancer, $\mathrm{AR}$ is a potential marker for metastatic prostate cancer. Crnalic et $a^{22}$ showed that only two cases out of 58 investigated bone metastases were negative for AR. Concordant with these findings, we also observed a high-expression frequency for $\mathrm{AR}$ in distant metastases (91\%). Furthermore, we found an increased AR expression from benign prostatic tissue to metastatic prostate cancer. However, other studies that only included lymph node metastases showed a lower or constant level of AR compared with primary prostate cancer. ${ }^{24-26}$

Notably, expression of PSA, PSAP, and AR is not restricted to prostatic tissue. ${ }^{5,6,27}$ High specificity is an essential requirement for diagnostic markers. Mhawech-Fauceglia et $a l^{10}$ investigated the expression of PSMA in non-prostatic tissue and concluded that apical PSMA expression is highly specific for prostate. PSMA expression was observed in other tumor entities; however, expression in these cases was restricted to the cytoplasm. ${ }^{10}$ Furthermore, PSMA is known to be highly expressed in metastatic prostate cancer. ${ }^{28,29}$ In our study, $91 \%$ of the lymph node and $94 \%$ of distant metastases displayed an expression of PSMA, which underlines the role as a potential diagnostic target. Other authors also reported a high sensitivity of $85-100 \%$ in lymph node and distant metastases. ${ }^{30,31}$

Until a few years ago, detection of TMPRSS2-ERG fusion was only possible using fluorescence in situ hybridization. Since TMPRSS2-ERG fusion highly correlates with ERG overexpression, the newly available monoclonal antibodies displaying ERG overexpression open up the opportunity for routine utilization. $^{11,32}$ ERG immunoreactivity was present in half of the primary prostate cancers being consistent with the frequency of TMPRSS2-ERG fusion and the findings of other immunohistochemical studies. ${ }^{11,33,34}$ We found an expression of ERG in approximately half of the lymph node metastases similar to the study of Hoogland et $a l^{33}$ with six out of nine lymph nodes expressing ERG. In distant metastases, fluorescence in situ hybridization analyses have shown an occurrence of TMPRSS2$E R G$ rearrangements in $25-35 \% .{ }^{12,15}$ We are among the first to investigate the protein level of ERG in metastases and could show that only $30 \%$ of the distant metastases cases were positive. In order to verify these results, larger sample sizes are necessary. However, it is worth mentioning that the TMPRSS2$E R G$ fusion is specific to prostate cancer ${ }^{12}$ and thus, an ERG protein expression in any epithelial cancer of unkown primary lesion is evidentiary for prostatic origin.

In summary, we are the first to comprehensively investigate the expression profile of widely used 

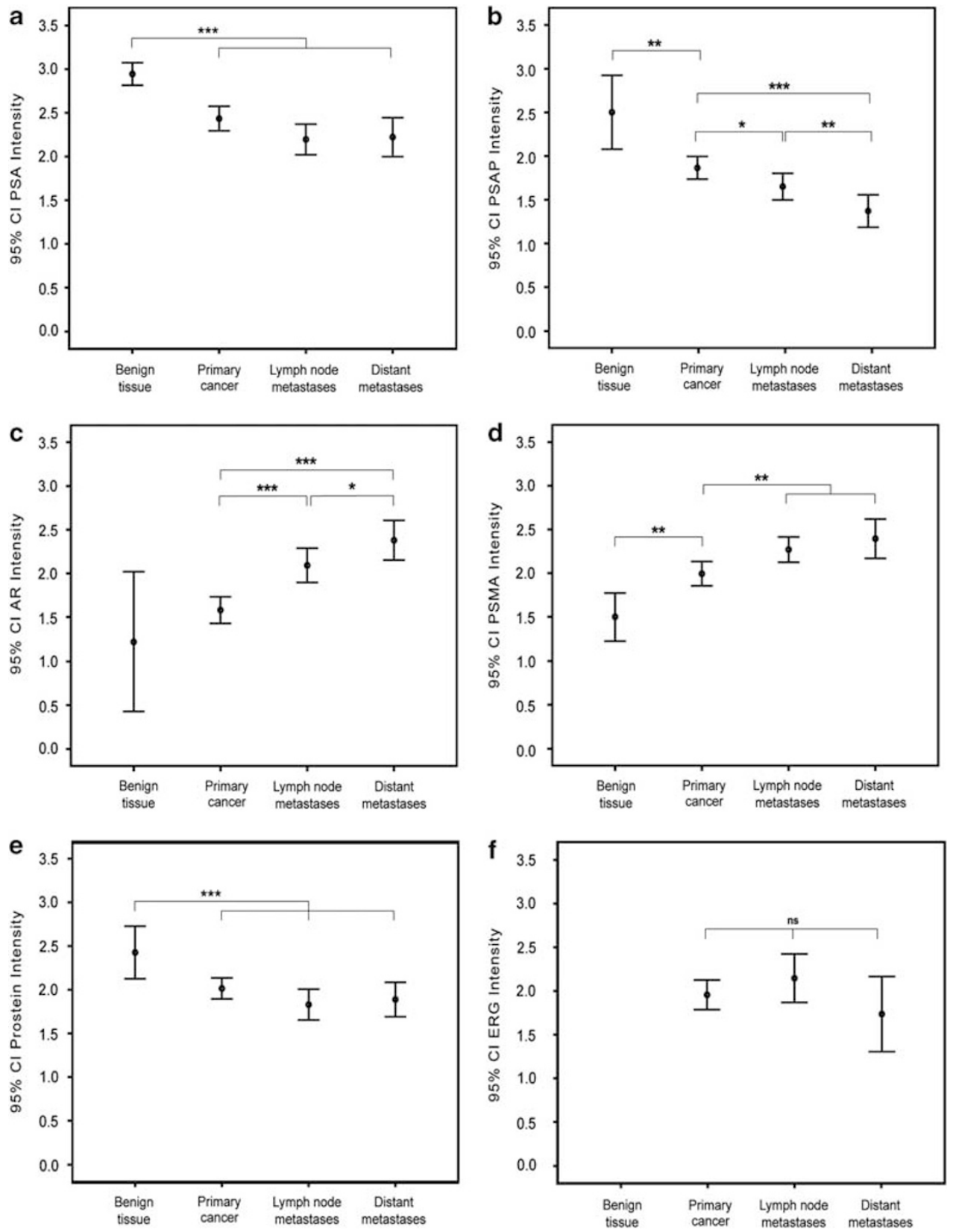

Figure 2 Staining intensity of PSA, PSAP, AR, PSMA, prostein, and ERG in normal prostatic tissue and neoplastic prostate tissue. PSA is significantly strongly expressed in benign prostatic tissue as compared with neoplastic tissue (a). PSAP expression decreases significantly from benign prostatic tissue to primary prostate cancer, lymph node metastases, and distant metastases (b). AR expression is weakly expressed in benign prostatic tissue but increases significantly from primary prostate cancer to metastatic prostate cancer (c). PSMA is moderately expressed in benign prostatic tissue but increases significantly in primary prostate cancer and metastatic prostate cancer (d). Prostein expression is significantly lower in malignant tissue than in benign prostatic tissue (e). ERG expression does not differ between primary prostate cancer and metastases (f). Only positive cases were included in the analyses; 1: low expression, 2: moderate expression, 3: strong expression.

diagnostic markers in metastatic prostate cancer. All markers except ERG were able to detect at least $70 \%$ of the lymph node metastases and distant metastases. Notably, all the distant metastases included in this study were treated with hormone ablation prior to the biopsy/extraction, which might lead to a potential change of expression of those markers. Further studies are needed to clarify this issue. The data obtained from this study support the utility of
PSA as a diagnostic marker with a high sensitivity of $97 \%$ for distant metastases. Cases that were negative for PSA could be additionally detected either with antibodies against AR or prostein which increases the sensitivity up to $98 \%$ for lymph node metastases and $100 \%$ for distant metastases. A panel consisting of the specific markers PSMA and prostein reached the same sensitivity of $98 \%$ for lymph node metastases and $100 \%$ for distant metastases as the 
above-mentioned combinations. As PSMA and prostein are described as highly specific, we recommend a staining panel of those two markers to initially detect prostatic origin of a metastasis.

\section{Acknowledgments}

We thank the staff of the immunohistochemistry laboratory especially Susanne Steiner for their technical assistance. The study was supported by a grant from the German Research Foundation (Deutsche Forschungsgemeinschaft (DFG), Emmy-Noether-Program, (PE1179/2-1), the Rudolf-Becker-Foundation, and the Wilhelm-Sander-Foundation (2011.077.2), to SP and the German Cancer Aid (Mildred-Scheel Doctoral Student Fellowship Program) to SH. SD is supported by the Medical Faculty Heidelberg.

\section{Disclosure/conflict of interest}

The authors declare no conflict of interest.

\section{References}

1 Siegel R, Naishadham D, Jemal A. Cancer statistics, 2012. CA Cancer J Clin 2012;62:10-29.

2 Yin M, Dhir R, Parwani AV. Diagnostic utility of p501s (prostein) in comparison to prostate specific antigen (PSA) for the detection of metastatic prostatic adenocarcinoma. Diagn Pathol 2007;2:41.

3 Fizazi K, Greco FA, Pavlidis N, et al. Cancers of unknown primary site: ESMO clinical practice guidelines for diagnosis, treatment and follow-up. Ann Oncol 2011;22(Suppl 6):vi64-vi68.

4 Sheridan T, Herawi M, Epstein JI, et al. The role of P501S and PSA in the diagnosis of metastatic adenocarcinoma of the prostate. Am J Surg Pathol 2007;31: 1351-1355.

5 Carder PJ, Speirs V, Ramsdale J, et al. Expression of prostate specific antigen in male breast cancer. J Clin Pathol 2005;58:69-71.

6 Elgamal AA, Ectors NL, Sunardhi-Widyaputra S, et al. Detection of prostate specific antigen in pancreas and salivary glands: a potential impact on prostate cancer overestimation. J Urol 1996;156:464-468.

7 Genega EM, Hutchinson B, Reuter VE, et al. Immunophenotype of high-grade prostatic adenocarcinoma and urothelial carcinoma. Mod Pathol 2000;13:1186-1191.

8 Mhawech P, Uchida T, Pelte MF. Immunohistochemical profile of high-grade urothelial bladder carcinoma and prostate adenocarcinoma. Hum Pathol 2002; 33:1136-1140.

9 Kalos M, Askaa J, Hylander BL, et al. Prostein expression is highly restricted to normal and malignant prostate tissues. Prostate 2004;60:246-256.

10 Mhawech-Fauceglia $\mathrm{P}$, Zhang S, Terracciano L, et al. Prostate-specific membrane antigen (PSMA) protein expression in normal and neoplastic tissues and its sensitivity and specificity in prostate adenocarcinoma: an immunohistochemical study using mutiple tumour tissue microarray technique. Histopathology 2007;50: $472-483$
11 Braun M, Goltz D, Shaikhibrahim Z, et al. ERG protein expression and genomic rearrangement status in primary and metastatic prostate cancer-a comparative study of two monoclonal antibodies. Prostate Cancer Prostatic Dis 2012;15:165-169.

12 Scheble VJ, Braun M, Beroukhim R, et al. ERG rearrangement is specific to prostate cancer and does not occur in any other common tumor. Mod Pathol 2010;23:1061-1067.

13 Mosquera JM, Mehra R, Regan MM, et al. Prevalence of TMPRSS2-ERG fusion prostate cancer among men undergoing prostate biopsy in the United States. Clin Cancer Res 2009;15:4706-4711.

14 Tomlins SA, Rhodes DR, Perner S, et al. Recurrent fusion of TMPRSS2 and ETS transcription factor genes in prostate cancer. Science 2005;310:644-648.

15 Perner S, Mosquera JM, Demichelis F, et al. TMPRSS2ERG fusion prostate cancer: an early molecular event associated with invasion. Am J Surg Pathol 2007;31: 882-888.

16 Downes MR, Torlakovic EE, Aldaoud N, et al. Diagnostic utility of androgen receptor expression in discriminating poorly differentiated urothelial and prostate carcinoma. J Clin Pathol 2013;66:779-786.

17 Visakorpi $\mathrm{T}$, Hyytinen $\mathrm{E}$, Koivisto $\mathrm{P}$, et al. In vivo amplification of the androgen receptor gene and progression of human prostate cancer. Nat Genet 1995;9:401-406.

18 Kirschenbaum A, Liu XH, Yao S, et al. Prostatic acid phosphatase is expressed in human prostate cancer bone metastases and promotes osteoblast differentiation. Ann NY Acad Sci 2011;1237:64-70.

19 Goldstein NS. Immunophenotypic characterization of 225 prostate adenocarcinomas with intermediate or high Gleason scores. Am J Clin Pathol 2002;117:471-477.

20 Bostwick DG, Pacelli A, Blute M, et al. Prostate specific membrane antigen expression in prostatic intraepithelial neoplasia and adenocarcinoma: a study of 184 cases. Cancer 1998;82:2256-2261.

21 Gutman EB, Sproul EE, Gutman AB. Significance of increased phosphatase activity at the site of osteoblastic metastases secondary to carcinoma of the prostate gland. Am J Cancer 1936;485-495.

22 Crnalic S, Hornberg E, Wikstrom P, et al. Nuclear androgen receptor staining in bone metastases is related to a poor outcome in prostate cancer patients. Endocr Relat Cancer 2010;17:885-895.

23 Miyoshi Y, Ishiguro H, Uemura H, et al. Expression of AR associated protein 55 (ARA55) and androgen receptor in prostate cancer. Prostate 2003;56:280-286.

24 Tamburrino L, Salvianti F, Marchiani S, et al. Androgen receptor (AR) expression in prostate cancer and progression of the tumor: lessons from cell lines, animal models and human specimens. Steroids 2012;77:996-1001.

25 Fleischmann A, Rocha C, Schobinger S, et al. Androgen receptors are differentially expressed in Gleason patterns of prostate cancer and down-regulated in matched lymph node metastases. Prostate 2011;71: 453-460.

26 Sweat SD, Pacelli A, Bergstralh EJ, et al. Androgen receptor expression in prostate cancer lymph node metastases is predictive of outcome after surgery. J Urol 1999;161:1233-1237.

27 Graddis TJ, McMahan CJ, Tamman J, et al. Prostatic acid phosphatase expression in human tissues. Int J Clin Exp Pathol 2011;4:295-306. 
28 Chang SS, Reuter VE, Heston WD, et al. Comparison of anti-prostate-specific membrane antigen antibodies and other immunomarkers in metastatic prostate carcinoma. Urology 2001;57:1179-1183.

29 Perner S, Hofer MD, Kim R, et al. Prostate-specific membrane antigen expression as a predictor of prostate cancer progression. Hum Pathol 2007;38: 696-701.

30 Ananias HJ, van den Heuvel MC, Helfrich W, et al. Expression of the gastrin-releasing peptide receptor, the prostate stem cell antigen and the prostate-specific membrane antigen in lymph node and bone metastases of prostate cancer. Prostate 2009;69:1101-1108.

31 Mannweiler S, Amersdorfer $\mathrm{P}$, Trajanoski S, et al. Heterogeneity of prostate-specific membrane antigen
(PSMA) expression in prostate carcinoma with distant metastasis. Pathol Oncol Res: POR 2009;15:167-172.

32 Park K, Tomlins SA, Mudaliar KM, et al. Antibodybased detection of ERG rearrangement-positive prostate cancer. Neoplasia 2010;12:590-598.

33 Hoogland AM, Jenster G, van Weerden WM, et al. ERG immunohistochemistry is not predictive for PSA recurrence, local recurrence or overall survival after radical prostatectomy for prostate cancer. Mod Pathol 2012;25:471-479.

34 Teng LH, Wang C, Begin LR, et al. ERG protein expression and gene rearrangements are present at lower rates in metastatic and locally advanced castration-resistant prostate cancer compared to localized disease. Urology 2013;82:394-399. 